\title{
Serum Levels of Phosphorus, Parathyroid Hormone, and Calcium and Risks of Death and Cardiovascular Disease in Individuals With Chronic Kidney Disease A Systematic Review and Meta-analysis
}

\begin{tabular}{l} 
Suetonia C. Palmer, MB ChB, PhD \\
\hline Andrew Hayen, PhD \\
\hline Petra Macaskill, PhD \\
\hline Fabio Pellegrini, MSc \\
\hline Jonathan C. Craig, MB ChB, PhD \\
\hline Grahame J. Elder, MB BS, PhD \\
\hline Giovanni F. M. Strippoli, MD, PhD \\
LINICAL PRACTICE GUIDE- \\
lines are a powerful influ- \\
ence on management strate- \\
gies for common conditions.
\end{tabular} When evidence that treating risk factors reduces disease and improves health outcomes is provided by large and well-conducted randomized controlled trials, guidelines can appropriately summarize the relevant data and widely disseminate recommendations for best practice. However, when practice guidelines promote therapeutic strategies without sufficient evidence of effectiveness or harms, overtreatment and widespread inappropriate use of medications, services, or devices may occur. Accordingly, guidelines may recommend health interventions that do not actually improve population outcomes or the quality of care, unnecessarily increase health care expenditure, and may even harm individuals who might be expected to receive small or negligible treatment benefit.

An important example is anemia in chronic kidney disease. Guidelines ${ }^{1,2}$

For editorial comment see $p 1138$.

Context Clinical practice guidelines on the management of mineral and bone disorders due to chronic kidney disease recommend specific treatment target levels for serum phosphorus, parathyroid hormone, and calcium.

Objective To assess the quality of evidence for the association between levels of serum phosphorus, parathyroid hormone, and calcium and risks of death, cardiovascular mortality, and nonfatal cardiovascular events in individuals with chronic kidney disease.

Data Sources The databases of MEDLINE (1948 to December 2010) and EMBASE (1947 to December 2010) were searched without language restriction. Hand searches also were conducted of the reference lists of primary studies, review articles, and clinical guidelines along with full-text review of any citation that appeared relevant.

Study Selection Of 8380 citations identified in the original search, 47 cohort studies ( $\mathrm{N}=327644$ patients) met the inclusion criteria.

Data Extraction The characteristics of study design, participants, exposures, and covariates together with the outcomes of all-cause mortality, cardiovascular mortality, and nonfatal cardiovascular events at different levels of serum phosphorus, parathyroid hormone, and calcium were analyzed within studies. Data were summarized across studies (when possible) using random-effects meta-regression.

Data Synthesis The risk of death increased $18 \%$ for every $1-\mathrm{mg} / \mathrm{dL}$ increase in serum phosphorus (relative risk [RR], 1.18 [95\% confidence interval $\{\mathrm{Cl}\}, 1.12-1.25]$ ). There was no significant association between all-cause mortality and serum level of parathyroid hormone (RR per 100-pg/mL increase, 1.01 [95\% Cl, 1.00-1.02]) or serum level of calcium (RR per $1-\mathrm{mg} / \mathrm{dL}$ increase, 1.08 [95\% Cl, 1.00-1.16]). Data for the association between serum level of phosphorus, parathyroid hormone, and calcium and cardiovascular death were each available in only 1 adequately adjusted cohort study. Lack of adjustment for confounding variables was not a major limitation of the available studies.

Conclusions The evidentiary basis for a strong, consistent, and independent association between serum levels of calcium and parathyroid hormone and the risk of death and cardiovascular events in chronic kidney disease is poor. There appears to be an association between higher serum levels of phosphorus and mortality in this population.

JAMA. 2011;305(11):1119-1127

www.jama.com

Author Affiliations: Department of Medicine, University of Otago, Christchurch, New Zealand (Dr Palmer); Brigham and Women's Hospital, Harvard Medical School, Boston, Massachusetts (Dr Palmer); School of Public Health, University of Sydney, Sydney, Australia (Drs Hayen, Macaskill, Craig, and Strippoli); Department of Clinical Pharmacology and Epidemiology, Mario Negri Sud Consortium, S Maria Imbaro, Italy (Mr Pellegrini and $\mathrm{Dr}$ Strippoli); Scientific Institute Casa Sollievo della
Sofferenza, Italy (Mr Pellegrini); Cochrane Renal Group, Sydney, Australia (Drs Craig and Strippoli); Department of Renal Medicine, Westmead Hospital and the Bone and Mineral Program, Garvan Institute of Medical Research, Sydney, Australia (Dr Elder); and Medical Scientific Office, Diaverum, Lund, Sweden (Dr Strippoli).

Corresponding Author: Giovanni F. M. Strippoli, MD, PhD, Consorzio Mario Negri Sud, Via Nazionale 8/a, 66030 S. Maria Imbaro, Italy (strippoli@negrisud.it). 
promoted anemia as a risk factor for mortality based on observational evidence of an association between low hemoglobin levels and the risks of death and hospitalization. ${ }^{3,4}$ It was argued that anemia required treatment in individuals with chronic kidney disease, but randomized controlled trials testing epoetins (an intervention that increases hemoglobin) showed that targeting higher hemoglobin levels caused harm, ${ }^{5-7}$ indicating that existing guidelines (based on observational data) were at best misleading.

A similar pattern has emerged in guidelines relating to the management of bone and mineral disorders in individuals with chronic kidney disease. Chronic kidney disease causes complicated changes in calcium and phosphorus metabolism ${ }^{8}$ for which numerous drugs including phosphorus binders, vitamin D compounds, and calcimimetic agents have been specifically developed and promoted. Nephrology guidelines recommend targets and treatment strategies to correct serum levels of phosphorus, calcium, and parathyroid hormone because observational data suggest there is an association between these potential risk biomarkers and vascular disease and death. ${ }^{9,10}$ However, to date, randomized controlled trials have not shown that treating mineral levels with existing treatment options reduces cardiovascular events or mortality. ${ }^{11-13}$ Nearly all such trials have been underpowered for mortality and cardiovascular outcomes, and a large comparative trial of phosphorus binders in hemodialysis found no evidence for benefit on mortality. ${ }^{14}$ Existing guidelines (based primarily on observational studies) may be inappropriately promoting treatment for abnormal mineral metabolism in individuals with chronic kidney disease.

Given the burden of cardiovascular morbidity in individuals with chronic kidney disease and the potential for serum levels of phosphorus, parathyroid hormone, and calcium to act as important modifiable risk factors for disease, we critically evaluated the evi- dentiary basis for an association between serum mineral levels and cardiovascular disease and mortality in individuals with chronic kidney disease. In particular, we sought to disaggregate those studies with more valid estimates of the association between mineral levels and outcomes through adjustment for known confounding variables.

\section{METHODS}

We performed a systematic review and meta-analysis of cohort studies measuring the association between serum levels of phosphorus, parathyroid hormone, or calcium and death or cardiovascular events in individuals with chronic kidney disease according to the Preferred Reporting Items for Systematic Reviews and Meta-Analyses guidelines. ${ }^{15}$ A specialized librarian conducted a systematic and comprehensive database search of MEDLINE (1948 to December 2010) and EMBASE (1947 to December 2010) using a strategy that combined text word and Medical Subject Headings without language restriction (eTable 1 at http://www.jama .com). We also hand-searched the reference lists of primary studies, review articles, and clinical guidelines. We inspected the full text of any citation that appeared relevant.

We extracted data on the characteristics of study design, participants, exposures, and covariates together with the outcomes of all-cause mortality, cardiovascular mortality, and nonfatal cardiovascular events. Whenever available, we extracted the number of individuals at risk, and the adjusted hazard ratio or relative risk (RR) per unit baseline serum concentrations of phosphorus $(1 \mathrm{mg} / \mathrm{dL})$, parathyroid hormone $(100 \mathrm{pg} / \mathrm{mL})$, or calcium $(1 \mathrm{mg} /$ $\mathrm{dL})$. The standard error of the estimate was also extracted or estimated from the reported 95\% confidence interval (CI) if necessary. In addition, we extracted the adjusted hazard ratio or RR for each reported category of baseline serum concentrations of phosphorus, parathyroid hormone, or calcium. For each category of serum level exposure, we assigned the numerical value for the risk category as the midpoint of the upper and lower bound of the reported category. ${ }^{16}$ Whenever the category was open ended at the upper bound (eg, serum phosphorus $>11 \mathrm{mg} / \mathrm{dL}$ ), the assigned value recorded was the lower bound value of the category $(11 \mathrm{mg} /$ dL). Risk estimates for discrete patient cohorts reported within the same study (ie, participants receiving hemodialysis or peritoneal dialysis) were extracted separately. ${ }^{17,18}$ Whenever more than 1 publication reported data, we used all available reports to inform our data extraction while taking care to avoid duplication of information. Risks of bias in each study were assessed according to previously published methods. ${ }^{19}$

We plotted the reported log RR estimate for the outcomes of all-cause mortality, cardiovascular mortality, or nonfatal cardiovascular events against the corresponding category of serum level of phosphorus, parathyroid hormone, and calcium in each available cohort to qualitatively assess the linearity of the associations on the log scale. We then used the study-specific estimates of the RR per unit increase in serum level to calculate the summary estimates of RR (with 95\% CI) per unit of increase in serum level of phosphorus, parathyroid hormone, or calcium. When this estimate was not available for a study, we used the methods described by Hartemink et $\mathrm{al}^{20}$ to calculate RR estimates per unit of exposure based on the category-specific estimates of risk if the required data were reported. This method assumes that the relationship between the RR estimate per unit of exposure (level of serum phosphorus, parathyroid hormone, or calcium) is approximately linear.

Using studies where a linear association between log RR and exposure was reported or was estimable, we fitted a random-effects meta-regression model using the log RR per unit of exposure as the outcome to obtain a summary estimate of the log RR. The incremental units assigned were serum levels of phosphorus per $1-\mathrm{mg} / \mathrm{dL}$ in- 
crease relative to a referent value of 3.5 $\mathrm{mg} / \mathrm{dL}$ (to convert to $\mathrm{mmol} / \mathrm{L}$, multiply by 0.323 ), parathyroid hormone per $100-\mathrm{pg} / \mathrm{mL}$ increase relative to a referent value of $200 \mathrm{pg} / \mathrm{mL}$ (to convert to $\mathrm{ng} / \mathrm{L}$, multiply by 1 ), and calcium per $1-\mathrm{mg} / \mathrm{dL}$ increase relative to a referent serum value of $8.4 \mathrm{mg} / \mathrm{dL}$ (to convert to $\mathrm{mmol} / \mathrm{L}$, multiply by 0.25 ). The meta-regression took account of the standard error for the estimate for each study. The summary log RR was exponentiated to obtain the summary estimate of RR.

We used subgroup analysis and meta-regression to assess the effect of adjustment for the key covariates of age, cardiovascular disease, diabetes status, time receiving dialysis (or estimated glomerular filtration rate for individuals not requiring dialysis), and race or ethnicity. The quality of adjustment was defined a priori as adequate when outcomes were adjusted for all 5 covariates and as partial when adjusted for 4 or fewer covariates. We also assessed the RR of mortality per unit of exposure for each of the following study-level covariates (when possible): age, proportion of men, race, stage of chronic kidney disease (dialysis, transplant, chronic kidney disease), dialysis adequacy, date of publication, duration of study, exposure measurement, and number of participants.

We conducted sensitivity analyses to evaluate whether using adjustment for cardiovascular disease as a criterion for assessing study quality altered our findings. Also, several studies were derived from the same data sources (eg, US Renal Data System, Dialysis Outcomes Practice Patterns Study). Whenever possible, we conducted sensitivity analyses by including studies from the same data source separately in summary analyses. For all analyses, a 2 -tailed $P$ value of less than .05 indicates statistical significance. Analyses were undertaken using SAS software version 9.2 (SAS Institute Inc, Cary, North Carolina) and Stata software version 11 (StataCorp, College Station, Texas) for the meta-regression.

\section{RESULTS}

A systematic search yielded 47 eligible studies $(\mathrm{N}=327644)$ in 49 cohorts of adults with chronic kidney disease (FIGURE 1; eTable 2 at http: //www.jama.com). The population sizes of the studies, all published between 1995 and 2010, varied between 99 and 78420 individuals. The 12 studies that enrolled more than 5000 individuals were derived from registry databases. ${ }^{21-32}$ Study duration ranged be-

Figure 1. Flow Diagram of the Identification Process for Eligible Studies

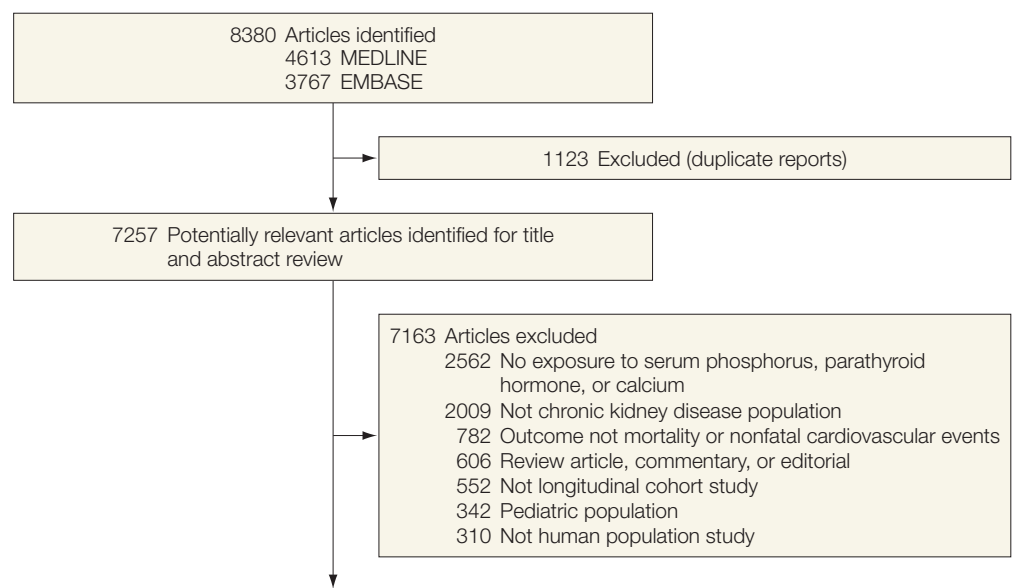

94 Articles identified for full-text review

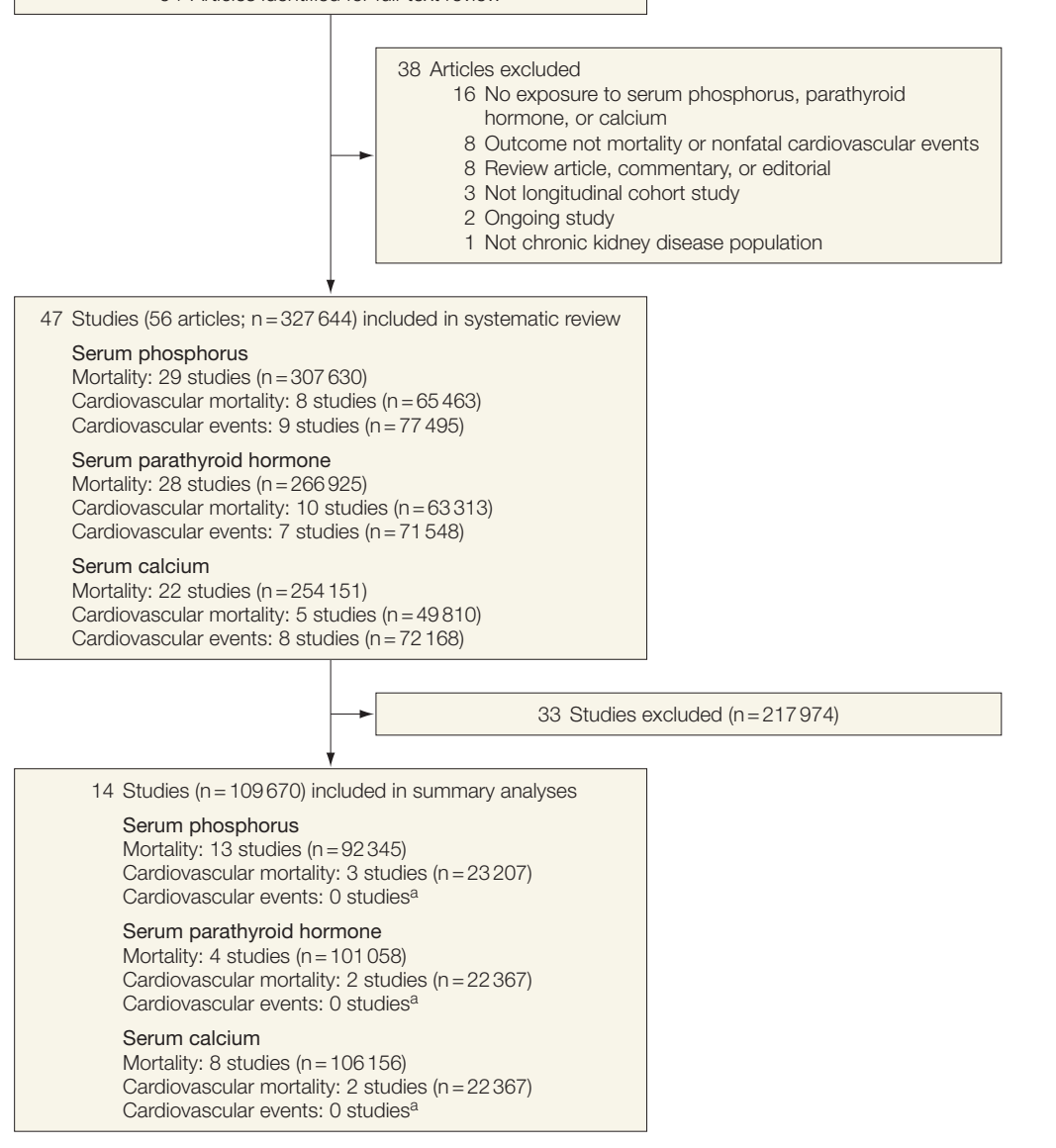

asummary estimate not possible due to insufficient data. 
tween 12 and 360 months. The vast majority of data were derived from cohorts requiring dialysis. Twenty-four studies (51\%) assessed outcomes in 310273 individuals receiving hemodialysis, ${ }^{21-44}$ and 3 studies assessed outcomes in 1124 participants receiving peritoneal dialysis. ${ }^{45-47}$ Five studies combined analysis in 3233 participants receiving hemodialysis and peritoneal dialysis, ${ }^{48-52}$ and 2 studies $(n=2251)$ analyzed outcomes for separate cohorts receiving hemodialysis and peritoneal dialysis. ${ }^{17,18}$ One study combined 98 individuals receiving dialysis, transplant recipients, and those with chronic kidney disease. ${ }^{53}$ Four studies evaluated 1675 kidney transplant recipients. ${ }^{54-57}$ Eight studies $(n=8990)$ included individuals with chronic kidney disease in stages 3 through $5 .^{58-65}$
Outcomes were evaluated based on serum exposures at commencement of dialysis in 8 cohorts, ${ }^{18,38,41,43,48-51}$ at the time of kidney transplantation in 3 cohorts, ${ }^{55,56,62}$ and at the onset of chronic kidney disease in 2 cohorts. ${ }^{63,64}$ The definitions and sources of data for all-cause mortality, cardiovascular mortality, and cardiovascular events that were reported in the included studies appear in eTable 3 (at http://www.jama.com). Car-

Figure 2. Risks of All-Cause Mortality Grouped According to Level of Study Adjustment for Confounding Variables

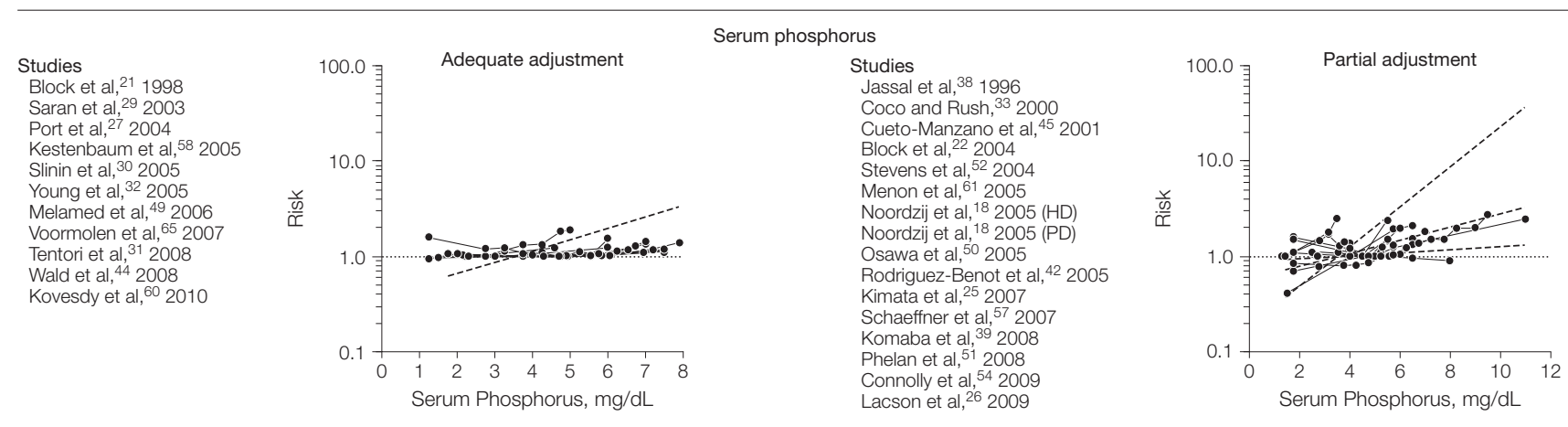

\begin{abstract}
Studies
Block et al, ${ }^{21} 1998$

Slinin et al, ${ }^{30} 2005$

Young et al, 322005

Melamed et al, 492006

Tentori et al, 312008

Dukkipati et al,, 352010
\end{abstract}
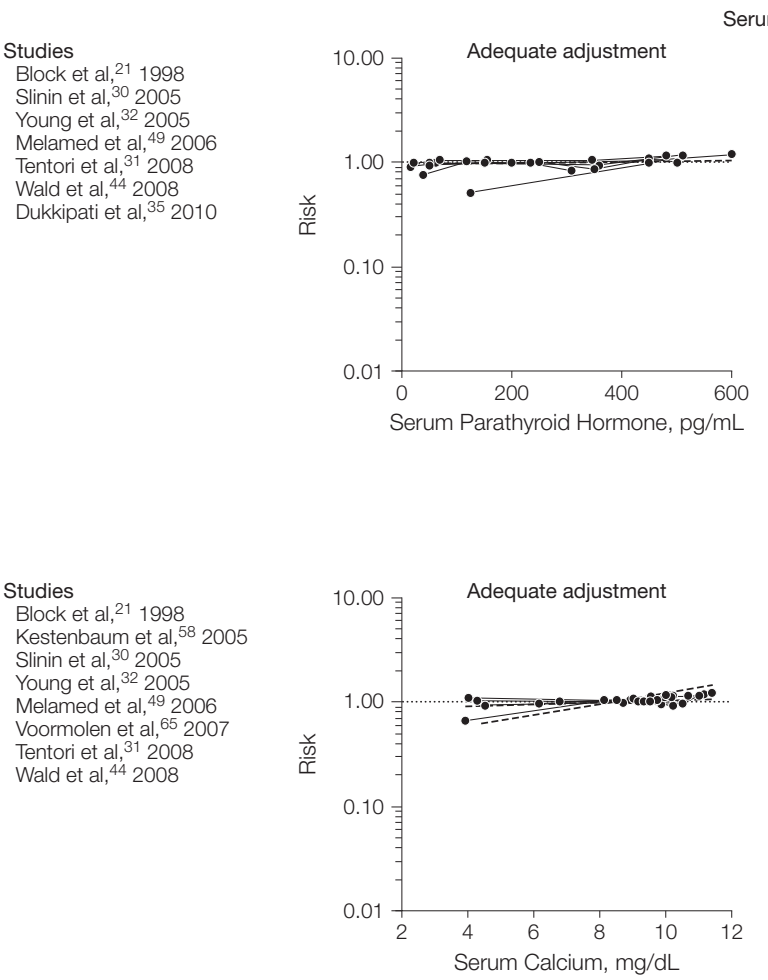

Serum calcium

Studies

Stevens et al,, 22004

Noordzij et al, 182005 (HD)

Noordzij et al, ${ }^{18} 2005$ (PD)

Osawa et al, 502005

Rodriquez-Benot et al 422005

Egbuna ot al 552007

Egbuna et al, 252007

Schaeffner et al, ${ }^{57} 2007$

Komaba et al ${ }^{39} 2008$

Lacson Jr et al, ${ }^{26} 2009$
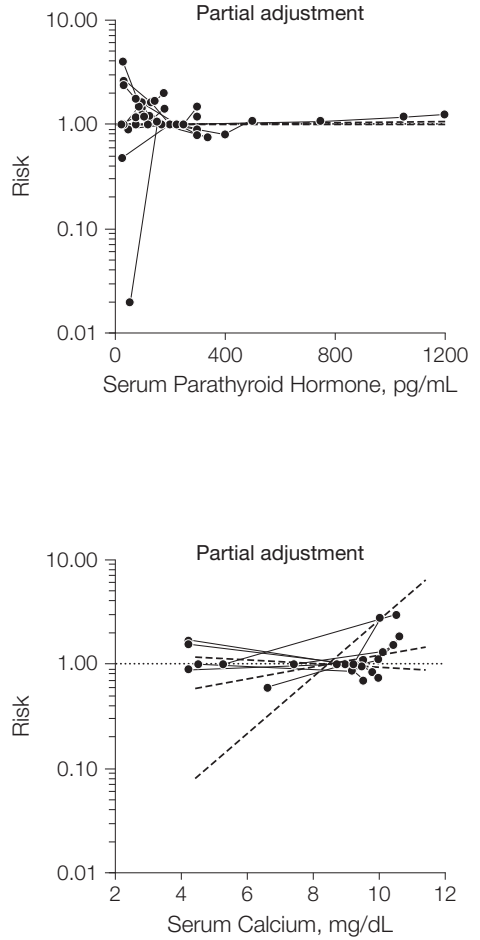

The data with dashed lines represent the studies graphically displayed that reported relative risk (RR) per unit change in serum phosphorus, parathyroid hormone, or calcium as continuous and not categorical variables (ie, these studies reported RR $\times$ per unit change, and their slope is provided in the graphs). Studies are stratified into adequate adjustment for confounding variables (all 5 covariates: age, race, time receiving dialysis [or estimated glomerular filtration rate], cardiovascular disease, and diabetes mellitus) or partial adjustment ( $<5$ covariates). HD refers to the hemodialysis cohort within the study. PD refers to the peritoneal dialysis cohort within the study. 
diovascular mortality and cardiovascular events were generally defined as death or hospitalization due to myocardial infarction, atherosclerotic heart disease, cardiac arrest, or arrhythmia.

By current standards, ${ }^{19}$ we found that reporting of study methods was incomplete, suggesting risks of bias existed in the included studies (eFigure 1 at http: //www.jama.com). Notably, data reporting completeness to follow-up (study attrition) and prognostic factor measurement were incomplete or unclear in approximately $90 \%$ of studies. Sixteen studies (34\%) reported on methods used to adjudicate mortality events and, of these, 1 study reported formal adjudication procedures,${ }^{34}$ suggesting misclassification bias was possible in the remainder. Age was adjusted for in 46 studies (98\%) and diabetes mellitus (81\%), time receiving dialysis or duration of chronic kidney disease (66\%), cardiovascular disease (47\%), and race or ethnicity (47\%). Thirteen studies (28\%) reported risks of mortality or cardiovascular events according to the mean of serum levels.* From these studies, we extracted risks for outcomes based on the mean of serum levels in the period before study commencement in 6 studies, ${ }^{22,26,35,40,49,58}$ mean of serum levels during the entire follow-up in 6 studies, ${ }^{24,33,37,43,48,56}$ and the mean of serum values in the period immediately before death or study completion in 1 study. ${ }^{42}$

Of the 47 studies overall, graphical evaluation of the relationship between categories of serum exposure and risk was not possible in 7 studies $(n=74114)$ because risk estimates for levels of serum exposure and an estimate of variance were not reported in an extractable format or a linear estimate of risk per unit increase in exposure was not extractable. ${ }^{23,24,43,47,56,62,63}$ Overall, 33 studies $(\mathrm{n}=217974)$ were not included in the meta-regression because they did not report the relationship between log RR per unit exposure, or this was not esti-

*References 22, 24, 26, 33, 35, 37, 40, 42, 43, 48, $49,58,60$ mable from category-specific estimates. ${ }^{17,18,21-24,27-31,34-37,41,43-49,51,53-56,59,62-64}$

On visual inspection, we found that the associations between serum levels of phosphorus, calcium, and parathyroid hormone and risks of all-cause mortality and other vascular outcomes were more consistent in studies with adequate adjustment for confounding variables than those studies with partial adjustment (FIGURE 2; eFigure 2 and eFigure 3 at http://www .jama.com). It should be noted that in general within most of the included studies, reported RRs derived from a base multivariate model that usually included age, race, sex, diabetes, and kid- ney function or duration of dialysis were not substantially altered by adjustment for additional potential confounding variables. Summary estimates for risks of all-cause mortality, cardiovascular mortality, and nonfatal cardiovascular events are shown in FIGURE 3.

In 12 partially adjusted studies (13 cohorts), the risk of death was higher with increasing levels of serum phosphorus (Figure 2) $\dagger$ In 10 adequately adjusted studies, the relationship between serum phosphorus and all-

tReferences 18, 22, 25, 26, 39, 42, 45, 51, 52, 54, 57,61

Figure 3. Summary Estimates for Risks of All-Cause Mortality and Cardiovascular Mortality Associated With Levels of Serum Phosphorus, Parathyroid Hormone, and Calcium

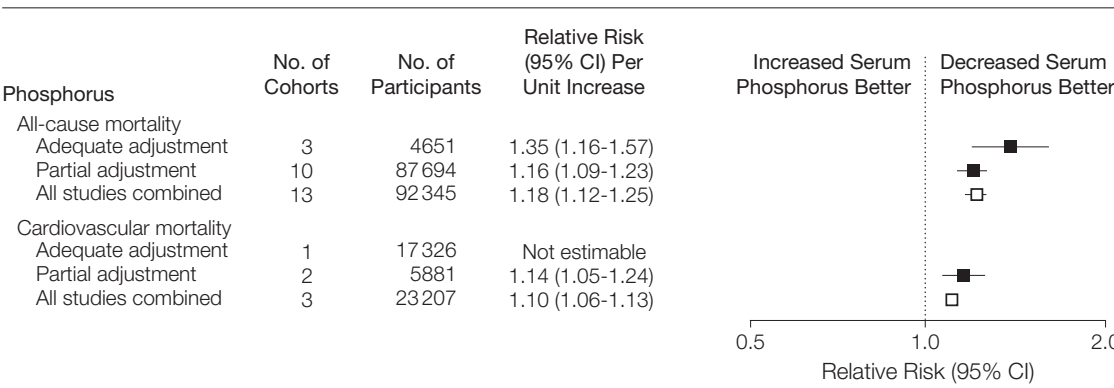

\begin{tabular}{|c|c|c|c|}
\hline Parathyroid hormone & $\begin{array}{l}\text { No. of } \\
\text { Cohorts }\end{array}$ & $\begin{array}{c}\text { No. of } \\
\text { Participants }\end{array}$ & $\begin{array}{l}\text { Relative Risk } \\
(95 \% \mathrm{Cl})\end{array}$ \\
\hline \multicolumn{4}{|l|}{ All-cause mortality } \\
\hline Adequate adjustment & 1 & 17326 & Not estimable \\
\hline Partial adjustment & 3 & 83732 & $1.01(0.99-1.03)$ \\
\hline All studies combined & 4 & 101058 & $1.01(1.00-1.02)$ \\
\hline \multicolumn{4}{|l|}{ Cardiovascular mortality } \\
\hline Adequa & 1 & 17326 & Not estimable \\
\hline Partial adjustment & 1 & 5041 & Not estimable \\
\hline All studies combined & 2 & 22367 & $1.05(0.99-1.11)$ \\
\hline
\end{tabular}

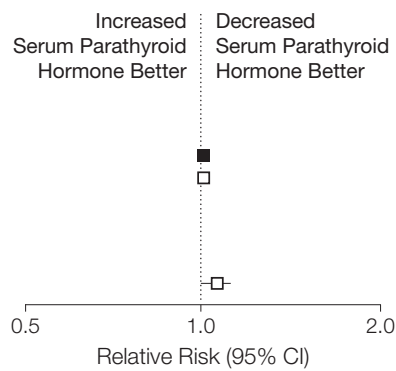

$\begin{array}{lcrc}\text { Calcium } & \begin{array}{c}\text { No. of } \\ \text { Cohorts }\end{array} & \begin{array}{c}\text { No. of } \\ \text { Participants }\end{array} & \begin{array}{c}\text { Relative Risk } \\ (95 \% \mathrm{Cl})\end{array} \\ \begin{array}{l}\text { All-cause mortality } \\ \text { Adequate adjustment }\end{array} & 2 & 22367 & 1.07(0.91-1.24) \\ \quad \begin{array}{c}\text { Partial adjustment } \\ \text { All studies combined }\end{array} & 6 & 83789 & 1.09(0.99-1.20) \\ \quad \text { Cardiovascular mortality } & 8 & 106156 & 1.08(1.00-1.16) \\ \quad \text { Adequate adjustment } & 1 & 17326 & \text { Not estimable } \\ \text { Partial adjustment } & 1 & 5041 & \text { Not estimable } \\ \text { All studies combined } & 2 & 22367 & 1.15(1.08-1.23)\end{array}$

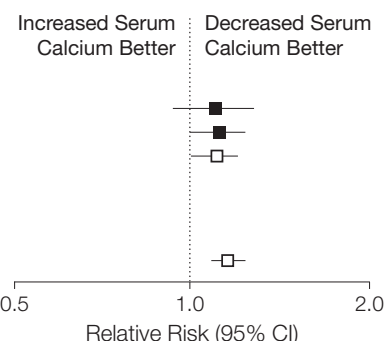

Risks of all-cause mortality, cardiovascular mortality, and nonfatal cardiovascular events are shown per 1-mg/dL increase in serum levels of phosphorus, $100-\mathrm{pg} / \mathrm{mL}$ increase in serum parathyroid hormone, and $1-\mathrm{mg} / \mathrm{dL}$ in crease in serum calcium. Summary estimates are not reported when only a single cohort contributed data. $\mathrm{Cl}$ indicates confidence interval. 
cause mortality was more consistent and an increasing risk of death was apparent at higher levels of serum phosphorus (>5.5 mg/dL). Based on studies reporting a continuous RR for each 1-mg/dL increase in serum phosphorus $^{25,26,33,38,40,42,50,52,58,60,61,65}$ together with a study in which the RR was estimated from reported event data, ${ }^{57}$ we found that for every $1-\mathrm{mg} / \mathrm{dL}$ increase in serum phosphorus, the risk of mortality increased by 35\% (RR, 1.35; 95\% $\mathrm{CI}, 1.16-1.57)$ in the 3 adequately adjusted studies $58,60,65$ and by $18 \%$ (RR, $1.18 ; 95 \% \mathrm{CI}, 1.12-1.25)$ in the 13 available studies overall. We could not include the study by Young et $\mathrm{al}^{32}$ in these analyses because there was strong indication of nonlinearity based on reported risks for categories of serum phosphorus (Figure 3). However, addition of this study using the reported RR per unit increase in serum phosphorus made a negligible difference to the summary estimates. Only a single study with adequate adjustment for confounders reported the association between serum level of phosphorus and risk of cardiovascular mortality $^{32}$; however, in the 3 studies reporting this outcome overall, the risk of cardiovascular mortality increased by $10 \%$ per $1-\mathrm{mg} / \mathrm{dL}$ increase in serum phosphorus (RR, 1.10; 95\% CI, 1.06-1.13). ${ }^{25,32,61}$ No data were available for the association between serum phosphorus and nonfatal cardiovascular events.

Whereas on visual inspection, 17 partially adjusted studies (19 cohorts) tended to show an increased risk of allcause mortality at lower serum levels of parathyroid hormone (Figure 2), $\ddagger$ a summary analysis of the 4 available studies ${ }^{25,26,32,50}$ found no relationship between parathyroid hormone and allcause mortality (Figure 3 ). Only a single adequately adjusted study reported the association between parathyroid hormone and cardiovascular mortality ${ }^{31}$ and no data were available for nonfatal cardiovascular events ${ }^{30}$ in a format

¥References 17, 18, 22, 25, 33, 34, 36, 37, 39, 41, $46,52,53,59,64$. extractable for analysis; therefore, summary estimates for these risks were not calculated. However, when we combined the adequately and partially adjusted studies, we found no evidence for association between parathyroid hormone and cardiovascular mortality (Figure 3).

No association between all-cause mortality and serum calcium levels was observed in 2 adequately adjusted studies (Figure 3). ${ }^{30-32,44,49}$ Only a single adequately adjusted study provided data for the association between serum calcium levels and risks of cardiovascular mortality ${ }^{32}$ and no data were available for nonfatal cardiovascular events. ${ }^{30}$ However, in all studies combined, there was evidence of association between serum calcium level and cardiovascular death $^{18,31}$ (RR, 1.15; 95\% CI, 1.081.23) (Figure 3). ${ }^{30,48}$

We performed univariate metaregression to identify other factors that might predict a relationship between serum level of phosphorus (eFigure 4 at http://www.jama.com) or calcium (eFigure 5) and risks of all-cause mortality. Univariate meta-regression was not possible for serum level of parathyroid hormone because fewer than 5 studies were available for the analyses. Risks of all-cause mortality associated with serum phosphorus and calcium were similar across different stages of chronic kidney disease. In the 4 studies $^{58,60,61,65}$ reporting outcomes for individuals with chronic kidney disease not yet requiring dialysis, the risk of allcause mortality for each $1-\mathrm{mg} / \mathrm{dL}$ increase in serum level of phosphorus (RR, 1.29; 95\% CI, 1.12-1.48) was similar to that observed in 8 studies $^{25,26,33,38,40,42,50,52}$ of individuals requiring dialysis (RR, 1.17 [95\% CI, 1.08$1.25] ; P=.22$ ). No evidence of an association between serum calcium and all-cause mortality was found in either individuals with earlier stages of chronic kidney disease (RR, 1.02; 95\% CI, $0.81-1.29)^{32,58}$ or those requiring dialysis (RR, 1.09 [95\% CI, 1.00-1.18]; $P=.63) .25,26,32,42,50,52,57$

We assessed the robustness of our assumption that adjusting for cardiovas- cular disease was necessary to define a study as adequately adjusted (in addition to age, race, duration of dialysis or kidney function, and presence of diabetes mellitus). When excluding cardiovascular disease as a criterion for adjustment quality, 6 studies ( 7 cohorts) were adjudicated as adequately adjusted. ${ }^{17,22,24,26,52,60}$ Of these, 2 studies $^{26,52}$ contributed data to the summary analysis for phosphorus and all-cause mortality. Categorizing these studies as adequately adjusted in the meta-analysis for this outcome did not alter the association we found between serum level of phosphorus and death (RR, 1.24; 95\% CI, 1.12-1.37). These 2 studies were also in the metaanalysis for calcium and all-cause mortality. Adjudication of these studies as adequate did not meaningfully alter the summary estimate we found for the association between serum level of calcium and all-cause mortality (RR, 1.09; 95\% CI, 0.98-1.21).

Several studies were derived from the same source; 4 studies were derived from the US Renal Data System ${ }^{21,23,28,30}$ and 5 studies included individuals sourced from the Dialysis Outcomes and Practice Patterns Study. ${ }^{25,27,29,31,32}$ Of all the meta-analyses, 2 studies from the same data source contributed to the summary estimates for parathyroid hormone and calcium and all-cause mortality. Entering these 2 studies $^{25,32}$ separately into the meta-analyses for all studies combined and only for adequately adjusted studies did not meaningfully change the associations we observed between serum parathyroid hormone and serum calcium with allcause mortality.

\section{COMMENT}

When receiving treatment designed to reach proposed serum target levels for phosphorus, parathyroid hormone, and calcium, individuals with chronic kidney disease, clinicians, and policy makers need to know whether serum levels have any evidence of association with health outcomes. Prior treatment trials for mineral and bone disorder in chronic kidney disease found 
no evidence for clinical benefit for any of the agents tested so far. ${ }^{11-13}$ Our study provides the first systematic and critical review using data from cohort studies of the association between serum levels of phosphorus, parathyroid hormone, and calcium and death and cardiovascular outcomes in individuals with chronic kidney disease.

Overall, we were unable to demonstrate any strong or consistent association between death and serum levels of parathyroid hormone and calcium in individuals with chronic kidney disease. While there may be an association between death and higher serum levels of phosphorus irrespective of the stage of kidney disease, high-quality data for an association between serum levels of phosphorus, parathyroid hormone, and calcium with cardiovascular mortality were available only in single cohort studies and summary estimates for cardiovascular events were not available. Consequently, based on the available cohort data and the absence of randomized controlled trials, the evidentiary basis for current clinical guideline-recommended targets of serum phosphorus, parathyroid hormone, and calcium in chronic kidney disease is poor. ${ }^{9,10}$

Broad adoption of health care practices that have insufficient evidence for safety or efficacy (in this case targeting serum mineral levels in individuals with chronic kidney disease) may lead to considerable unintended harm. Examples in medicine include the widespread adoption of treatment using encainide and flecainide to suppress ventricular tachyarrhythmias after myocardial ischemia and hormone therapy in postmenopausal women to prevent cardiovascular disease until randomized trials unequivocally demonstrated such interventions were harmful. ${ }^{66,67}$

We now demonstrate that the evidence is similarly lacking for serum mineral target levels as risk factors for poor health outcomes in individuals with chronic kidney disease despite nearly universal adoption of such targets in the field of nephrology ${ }^{68,69}$ The current data do not support the hypothesis that individuals with chronic kidney disease should have treatment to achieve targeted levels of serum parathyroid hormone or calcium to reduce mortality or cardiovascular morbidity, except at extreme levels in which hypocalcemia and hypercalcemia result in immediate, clinically apparent adverse events such as tetany and seizures. Furthermore, treating high phosphorus levels is linked to a substantial pill burden that is associated with lower quality of life in individuals with chronic kidney disease. ${ }^{70}$ While we do not conclude that normalizing serum levels of calcium or phosphorus or avoiding upper or lower extremes of serum level of parathyroid hormone is futile, high-quality evidence is required before specific treatment should be advocated strongly.

Accumulating cohort data demonstrate that associations exist between vitamin $\mathrm{D}$ and phosphorus binder treatment and lower mortality and cardiovascular events in individuals with chronic kidney disease that may be independent of their treatment effects on serum levels of phosphorus and parathyroid hormone.$^{71-74}$ Vitamin D receptor activation may attenuate left ventricle structural abnormalities, reduce left ventricle mass, and improve diastolic function. ${ }^{75}$ Given the associations between phosphorus level and cardiovascular events we found in the current study together with these uncontrolled data suggesting benefit for specific interventions in chronic kidney disease, we strongly advocate that large simple placebo-controlled randomized trials be conducted of vitamin D, calcimimetic agents (allosteric modulators of parathyroid calciumsensing receptors that lower parathyroid hormone secretion), or phosphorus binders in individuals with chronic kidney disease.

The earlier focus by the nephrology community on hemoglobin targets is a relevant cautionary tale. Because hemoglobin targets became the research priority, there was considerable delay in commencing a large pla- cebo-controlled trial for anemia in individuals with chronic kidney disease. Similarly, the limited evidentiary basis for an association between serum mineral levels and health outcomes in the present analysis suggests that target trials of serum levels of phosphorus, parathyroid hormone, and calcium should not be promoted in individuals with chronic kidney disease. ${ }^{76}$

Our study has limitations that should be considered. First, our conclusions are supported by low-quality data because summary effects are derived from uncontrolled cohort studies that are vulnerable to the unpredictable confounding effects of measured and unmeasured variables. Notably, within most of the included studies, RRs from a basic adjusted model were not altered by further adjustment for additional variables, indicating that partial adjustment was not an important limitation of these studies.

Second, we have assumed that the relationship between serum levels of phosphorus, parathyroid hormone, and calcium and health outcomes is approximately linear. While we assessed the validity of this assumption in individual studies using a regression analysis, the small number of exposure categories within most of the studies meant that we had limited power to detect nonlinear relationships between serum levels and log risks of mortality. Third, we have not assessed study data for the association between serum levels of alkaline phosphatase or vitamin D levels and mortality, which are both emerging as predictors of outcomes in populations with and without chronic kidney disease in cohort studies ${ }^{77-79}$ and are potential effect modifiers of the relationships between serum targets and outcomes that we have observed. We also have not studied the effects of serum mineral levels on other relevant clinical outcomes such as musculoskeletal pain or itch. Finally, phosphorus and calcium may function poorly as biomarkers because they represent only a fraction of body stores and have substantial intraindi- 
vidual variability over time (despite assay precision).

In conclusion, the evidence for an association between serum levels of phosphorus, calcium, or parathyroid hormone as risk factors for outcomes in individuals with chronic kidney disease (at any stage) is currently insufficient to inform clinical decision making, policy, or practice guidelines. Large placebo-controlled randomized trials of vitamin D compounds, phosphorus binders, and calcimimetic agents are now needed to evaluate whether treating mineral disorders improves health outcomes for individuals with chronic kidney disease.

Author Contributions: Drs Palmer and Strippoli had full access to all of the data in the study and take responsibility for the integrity of the data and the accuracy of the data analysis

Study concept and design: Palmer, Craig, Strippoli. Acquisition of data: Palmer, Strippoli.

Analysis and interpretation of data: Palmer, Hayen, Macaskill, Pellegrini, Craig, Elder, Strippoli.

Drafting of the manuscript: Palmer, Pellegrini, Craig, Strippoli.

Critical revision of the manuscript for important intellectual content: Palmer, Hayen, Macaskill, Craig, Elder, Strippoli.

Statistical analysis: Hayen, Macaskill, Pellegrini, Strippoli.

Obtained funding: Strippoli.

Administrative, technical, or material support: Strippoli. Study supervision: Craig, Strippoli.

Conflict of Interest Disclosures: All authors have completed and submitted the ICMJE Form for Disclosure of Potential Conflicts of Interest. Dr Palmer reported receiving assistance to travel for study supervision from the Amgen Dompe fellowship to the Consorzio Mario Negri Sud. Dr Elder reported being on the advisory boards of Amgen Australia and Shire Australia, being on the speakers bureaus for Amgen Australia, Genzyme Australia, Shire Australia, and Abbott Australia, and receiving a research grant from Roche Pharmaceuticals. No other disclosures were reported.

Funding/Support: Dr Palmer was the recipient of a Don and Lorraine Jacquot Fellowship from the Royal Australasian College of Physicians and received support from an unrestricted Amgen Dompé Consorzio Mario Negri Fellowship. Dr Macaskill reported receiving grant support administered through the University of Sydney to fund a program of research in screening and diagnostic testing from the Australian National Health and Medical Research Council.

Role of the Sponsors: The funding sources had no role in any stage of the design and conduct of the study; in the collection, management, analysis, and interpretation of data in the study; or in the preparation, review, or approval of the manuscript.

Online-Only Material: eTables 1-3 and eFigures 1-5 are available at http://www.jama.com.

\section{REFERENCES}

1. National Kidney Foundation Kidney Disease Outcomes Quality Initiative. Guidelines for anemia of chronic kidney disease: 2000. http://www.kidney.org /professionals/kdoqi/guidelines_updates/doqiupan_ii .html. Accessed February 6, 2011.
2. Revised European Best Practice Guidelines for the Management of Anaemia in Patients With Chronic Renal Failure: section II: targets for anaemia treatment. Nephrol Dial Transplant. 2004;19(suppl 2):ii6ii15.

3. Locatelli F, Conte F, Marcelli D. The impact of haematocrit levels and erythropoietin treatment on overall and cardiovascular mortality and morbidity. Nephrol Dial Transplant. 1998;13(7):1642-1644.

4. Ma JZ, Ebben J, Xia H, Collins AJ. Hematocrit level and associated mortality in hemodialysis patients. J Am Soc Nephrol. 1999;10(3):610-619.

5. Besarab A, Bolton WK, Browne JK, et al. The effects of normal as compared with low hematocrit values in patients with cardiac disease who are receiving hemodialysis and epoetin. N Engl J Med. 1998; 339(9):584-590.

6. Singh AK, Szczech L, Tang KL, et al; CHOIR Investigators. Correction of anemia with epoetin alfa in chronic kidney disease. $N$ Engl J Med. 2006; 355(20):2085-2098.

7. Pfeffer MA, Burdmann EA, Chen CY, et al. A trial of darbepoetin alfa in type 2 diabetes and chronic kidney disease. N Engl J Med. 2009;361(21):20192032

8. Moe SM, Drüeke T, Lameire N, et al. Chronic kidney disease-mineral-bone disorder. Adv Chronic Kidney Dis. 2007:14(1):3-12.

9. National Kidney Foundation. K/DOQI clinical practice guidelines for bone metabolism and disease in chronic kidney disease. Am J Kidney Dis. 2003; 42(4)(Suppl 3):S1-S201.

10. Kidney Disease: Improving Global Outcomes (KDIGO) CKD-MBD Work Group. KDIGO clinical practice guidelines for the diagnosis, evaluation, prevention, and treatment of chronic kidney diseasemineral and bone disorder (CKD-MBD). Kidney Int. 2009;76(suppl 113):S1-S130.

11. Navaneethan SD, Palmer SC, Craig JC, et al. Benefits and harms of phosphate binders in CKD. Am J Kidney Dis. 2009;54(4):619-637.

12. Palmer SC, McGregor DO, Macaskill P, et al. Metaanalysis: vitamin D compounds in chronic kidney disease. Ann Intern Med. 2007;147(12):840-853.

13. Strippoli GFM, Palmer SC, Tong A, et al. Metaanalysis of biochemical and patient-level effects of calcimimetic therapy. Am J Kidney Dis. 2006;47(5): 715-726.

14. Suki WN, Zabaneh R, Cangiano JL, et al. Effects of sevelamer and calcium-based phosphate binders on mortality in hemodialysis patients. Kidney Int. 2007; 72(9):1130-1137.

15. Liberati A, Altman DG, Tetzlaff J, et al. The PRISMA statement for reporting systematic reviews and meta-analyses of studies that evaluate health care interventions. Ann Intern Med. 2009;151 (4):W65-94.

16. Berlin JA, Longnecker MP, Greenland S. Metaanalysis of epidemiologic dose-response data. Epidemiology. 1993;4(3):218-228.

17. Avram MM, Sreedhara $R$, Fein $P$, et al. Survival on hemodialysis and peritoneal dialysis over 12 years with emphasis on nutritional parameters. Am J Kidney Dis. 2001;37(1)(suppl 2):S77-S80

18. Noordzij M, Korevaar JC, Boeschoten EW, et al. The kidney disease outcomes quality initiative (K/DOQI) guideline for bone metabolism and disease in CKD: association with mortality in dialysis patients. Am J Kidney Dis. 2005;46(5):925932.

19. Hayden JA, Côté P, Bombardier C. Evaluation of the quality of prognosis studies in systematic reviews. Ann Intern Med. 2006:144(6):427-437.

20. Hartemink N, Boshuizen HC, Nagelkerke NJ, et al. Combining risk estimates from observational studies with different exposure cutpoints. Am J Epidemiol. 2006:163(11):1042-1052.

21. Block GA, Hulbert-Shearon TE, Levin NW, Port
FK. Association of serum phosphorus and calcium $\times$ phosphate product with mortality risk in chronic hemodialysis patients. Am J Kidney Dis. 1998; 31(4):607-617.

22. Block GA, Klassen PS, Lazarus JM, et al. Mineral metabolism, mortality, and morbidity in maintenance hemodialysis. J Am Soc Nephrol. 2004;15 (8):2208-2218.

23. Ganesh SK, Stack AG, Levin NW, et al. Association of elevated serum $\mathrm{PO}(4), \mathrm{Ca} \times \mathrm{PO}(4)$ product, and parathyroid hormone with cardiac mortality risk in chronic hemodialysis patients. J Am Soc Nephrol. 2001;12(10):2131-2138.

24. Kalantar-Zadeh K, Kuwae N, Regidor DL, et al. Survival predictability of time-varying indicators of bone disease in maintenance hemodialysis patients. Kidney Int. 2006;70(4):771-780.

25. Kimata N, Albert JM, Akiba T, et al. Association of mineral metabolism factors with all-cause and cardiovascular mortality in hemodialysis patients [published correction appears in Hemodial Int. 2009:13(1):91]. Hemodial Int. 2007;11(3):340348.

26. Lacson E Jr, Wang W, Hakim RM, et al. Associates of mortality and hospitalization in hemodialysis. Am J Kidney Dis. 2009;53(1):79-90.

27. Port FK, Pisoni RL, Bragg-Gresham JL, et al. DOPPS estimates of patient life years attributable to modifiable hemodialysis practices in the United States. Blood Purif. 2004;22(1):175-180.

28. Rubel JR, Milford EL. The relationship between serum calcium and phosphate levels and cardiac valvular procedures in the hemodialysis population. Am J Kidney Dis. 2003;41(2):411-421.

29. Saran R, Bragg-Gresham JL, Rayner HC, et al. Nonadherence in hemodialysis. Kidney Int. 2003;64 (1):254-262.

30. Slinin Y, Foley RN, Collins AJ. Calcium, phosphorus, parathyroid hormone, and cardiovascular disease in hemodialysis patients. J Am Soc Nephrol. 2005; 16(6):1788-1793.

31. Tentori F, Blayney MJ, Albert JM, et al. Mortality risk for dialysis patients with different levels of serum calcium, phosphorus, and PTH. Am J Kidney Dis. 2008; 52(3):519-530

32. Young EW, Albert JM, Satayathum S, et al. Predictors and consequences of altered mineral metabolism. Kidney Int. 2005;67(3):1179-1187.

33. Coco M, Rush H. Increased incidence of hip fractures in dialysis patients with low serum parathyroid hormone. Am J Kidney Dis. 2000;36(6):11151121.

34. Drechsler C, Krane V, Grootendorst DC, et al. The association between parathyroid hormone and mortality in dialysis patients is modified by wasting. Nephrol Dial Transplant. 2009:24(10):3151-3157.

35. Dukkipati R, Kovesdy CP, Colman S, et al. Association of relatively low serum parathyroid hormone with malnutrition. J Ren Nutr. 2010;20(4):243254.

36. Dussol B, Morand P, Martinat C, et al. Influence of parathyroidectomy on mortality in hemodialysis patients. Ren Fail. 2007:29(5):579-586.

37. Guh JY, Chen HC, Chuang HY, et al. Risk factors and risk for mortality of mild hypoparathyroidism in hemodialysis patients. Am J Kidney Dis. 2002; 39(6):1245-1254

38. Jassal SV, Douglas JF, Stout RW. Prognostic markers in older patients starting renal replacement therapy. Nephrol Dial Transplant. 1996;11(6):1052-1057.

39. Komaba H, Igaki N, Takashima $M$, et al. Calcium, phosphorus, cardiovascular events and allcause mortality in hemodialysis patients. Ther Apher Dial. 2008;12(1):42-48

40. Marco MP, Craver L, Betriu A, et al. Higher impact of mineral metabolism on cardiovascular mortality in a European hemodialysis population. Kidney Int Suppl. 2003;(85):S111-S114. 
41. Morrone LF, Mazzaferro S, Russo D, et al. Interaction between parathyroid hormone and the Charlson comorbidity index on survival of incident haemodialysis patients. Nephrol Dial Transplant. 2009; 24(9):2859-2865.

42. Rodriguez-Benot $A$, Martin-Malo A, Alvarez-Lara $M A$, et al. Mild hyperphosphatemia and mortality in hemodialysis patients. Am J Kidney Dis. 2005; 46(1):68-77.

43. Spiegel DM, Raggi P, Smits G, Block GA. Factors associated with mortality in patients new to haemodialysis. Nephrol Dial Transplant. 2007; 22(12):3568-3572.

44. Wald R, Sarnak MJ, Tighiouart $H$, et al. Disordered mineral metabolism in hemodialysis patients. Am Kidney Dis. 2008:52(3):531-540.

45. Cueto-Manzano AM, Quintana-Piña E, Correa-Rotter R. Long-term CAPD survival and analysis of mortality risk factors. Perit Dial Int. 2001; 21(2):148-153.

46. Dimkovic NB, Bargman J, Vas S, Oreopoulos DG. Normal or low initial PTH levels are not a predictor of morbidity/mortality in patients undergoing chronic peritoneal dialysis. Perit Dial Int. 2002;22(2):204210.

47. Hakemi MS, Golbabaei M, Nassiri A, et al. Predictors of patient survival in continuous ambulatory peritoneal dialysis. Iran J Kidney Dis. 2010;4(1): 44-49.

48. Foley RN, Parfrey PS, Harnett JD, et al. Hypocalcemia, morbidity, and mortality in end-stage renal disease. Am J Nephrol. 1996;16(5):386393

49. Melamed $M L$, Eustace JA, Plantinga $L$, et al. Changes in serum calcium, phosphate, and PTH and the risk of death in incident dialysis patients. Kidney Int. 2006:70(2):351-357.

50. Osawa Y, Aoike I, Sakurabayashi T, et al. Serum calcium levels at first renal replacement therapy and patients survival. Clin Calcium. 2005;15(suppl 1): 167-172.

51. Phelan PJ, O'Kelly P, Walshe JJ, et al. The importance of serum albumin and phosphorous as predictors of mortality in ESRD patients. Ren Fail. 2008; 30(4):423-429.

52. Stevens LA, Djurdjev O, Cardew S, et al. Calcium, phosphate, and parathyroid hormone levels in combination and as a function of dialysis duration predict mortality. J Am Soc Nephrol. 2004;15(3):770779

53. Fellah $\mathrm{H}$, Hammami $M B$, Feki $M$, et al. Predictors for cardiovascular morbidity and overall mortality in Tunisian ESRD patients. Clin Biochem. 2009;42 $(7-8): 648-653$
54. Connolly GM, Cunningham R, McNamee PT, et al. Elevated serum phosphate predicts mortality in renal transplant recipients. Transplantation. 2009;87 (7):1040-1044

55. Egbuna OI, Taylor JG, Bushinsky DA, Zand MS. Elevated calcium phosphate product after renal transplantation is a risk factor for graft failure. Clin Transplant. 2007;21(4):558-566.

56. Krüger B, Schnitzbauer AA, Böger CA, et al. Pretransplant calcium levels have no predictive value for delayed graft function, long-term graft function, cardiovascular events, or graft and patient survival in renal transplantation. Transplant Proc. 2006;38(3): 697-700.

57. Schaeffner ES, Födinger $M$, Kramar R, et al. Prognostic associations of serum calcium, phosphate and calcium phosphate concentration product with outcomes in kidney transplant recipients. Transpl Int. 2007; 20(3):247-255

58. Kestenbaum B, Sampson JN, Rudser KD, et al. Serum phosphate levels and mortality risk among people with chronic kidney disease. J Am Soc Nephrol. 2005; 16(2):520-528

59. Kovesdy CP, Ahmadzadeh S, Anderson JE, et al. Secondary hyperparathyroidism is associated with higher mortality in men with moderate to severe chronic kidney disease. Kidney Int. 2008;73(11): 1296-1302

60. Kovesdy CP, Anderson JE, Kalantar-Zadeh K. Outcomes associated with serum phosphorus level in males with non-dialysis dependent chronic kidney disease. Clin Nephrol. 2010;73(4):268-275.

61. Menon V, Greene T, Pereira AA, et al. Relationship of phosphorus and calcium-phosphorus product with mortality in CKD. Am J Kidney Dis. 2005; 46(3):455-463

62. Roodnat I, van Gurp EA, Mulder PG, et al. High pretransplant parathyroid hormone levels increase the risk for graft failure after renal transplantation. Transplantation. 2006;82(3):362-367.

63. Smith DH, Johnson ES, Thorp ML, et al. Outcomes predicted by phosphorous in chronic kidney disease. Nephrol Dial Transplant. 2010;25(1):166174.

64. Smith DH, Johnson ES, Thorp ML, et al. Hyperparathyroidism in chronic kidney disease. J Bone Miner Metab. 2009;27(3):287-294.

65. Voormolen N, Noordzij M, Grootendorst DC et al. High plasma phosphate as a risk factor for decline in renal function and mortality in pre-dialysis patients. Nephrol Dial Transplant. 2007;22(10): 2909-2916.

66. Rossouw JE, Anderson GL, Prentice RL, et al. Risks and benefits of estrogen plus progestin in healthy post- menopausal women. JAMA. 2002;288(3):321333.

67. The Cardiac Arrhythmia Suppression Trial (CAST) Investigators. Preliminary report: effect of encainide and flecainide on mortality in a randomized trial of arrhythmia suppression after myocardial infarction. NEng/ J Med. 1989;321(6):406-412.

68. Lebner AC, Beard KM, Soroka SD, et al. Interprovincial differences in the achievement of K/DOQ targets of mineral metabolism in Canada. Nephrol Dial Transplant. 2011;26(1):156-163.

69. Blayney MJ, Tentori F. Trends and consequences of mineral bone disorder in haemodialysis patients. J Ren Care. 2009;35(suppl 1):7-13.

70. Chiu YW, Teitelbaum I, Misra M, et al. Pill burden, adherence, hyperphosphatemia, and quality of life in maintenance dialysis patients. Clin J Am Soc Nephrol. 2009;4(6):1089-1096.

71. Isakova $T$, Gutiérrez $O M$, Chang $Y$, et al. Phosphorus binders and survival on hemodialysis. J Am SoC Nephrol. 2009;20(2):388-396

72. St Peter WL, Li S, Liu J, et al. Effects of monthly dose and regular dosing of intravenous active vitamin D use on mortality among patients undergoing hemodialysis. Pharmacotherapy. 2009;29(2):154164.

73. Sugiura S, Inaguma D, Kitagawa A, et al. Administration of alfacalcidol for patients with predialysis chronic kidney disease may reduce cardiovascular disease events. Clin Exp Nephrol. 2010;14(1):4350.

74. Teng $M$, Wolf $M$, Ofsthun $M N$, et al. Activated injectable vitamin $D$ and hemodialysis survival. J Am Soc Nephrol. 2005;16(4):1115-1125.

75. Bodyak N, Ayus JC, Achinger S, et al. Activated vitamin $D$ attenuates left ventricular abnormalities induced by dietary sodium in Dahl salt-sensitive animals. Proc Natl Acad Sci U S A. 2007:104(43):1681016815.

76. Block GA, Persky MS, Ketteler M, et al. A randomized double-blind pilot study of serum phosphorus normalization in chronic kidney disease. Hemodial Int. 2009;13(3):360-362.

77. Kilkkinen A, Knekt P, Aro A, et al. Vitamin D status and the risk of cardiovascular disease death. Am Epidemiol. 2009;170(8):1032-1039.

78. Mehrotra R, Kermah DA, Salusky IB, et al. Chronic kidney disease, hypovitaminosis $\mathrm{D}$, and mortality in the United States. Kidney Int. 2009;76(9):977 983.

79. Tonelli $M$, Curhan G, Pfeffer $M$, et al. Relation between alkaline phosphatase, serum phosphate, and all-cause or cardiovascular mortality. Circulation. 2009; 120(18):1784-1792. 